\title{
Two-stage mechanism for activation of the DNA replication checkpoint kinase Cds1 in fission yeast
}

\author{
Yong-jie $\mathrm{Xu}^{1,2}$ Matthew Davenport, ${ }^{1}$ and Thomas J. Kelly ${ }^{1,3}$ \\ ${ }^{1}$ Program in Molecular Biology, Sloan-Kettering Institute, New York, New York 10021, USA; ${ }^{2}$ Biochemistry, Cellular \\ and Molecular Biology Program, Johns Hopkins School of Medicine, Baltimore, Maryland 21205, USA
}

The DNA replication checkpoint is a complex signal transduction pathway, present in all eukaryotic cells, that functions to maintain genomic integrity and cell viability when DNA replication is perturbed. In Schizosaccharomyces pombe the major effector of the replication checkpoint is the protein kinase Cds1. Activation of Cds1 is known to require the upstream kinase Rad3 and the mediator Mrc1, but the biochemical mechanism of activation is not well understood. We report that the replication checkpoint is activated in two stages. In the first stage, Mrc1 recruits Cds1 to stalled replication forks by interactions between the FHA domain of Cds1 and specific phosphorylated Rad3 consensus sites in Mrc1. Cds1 is then primed for activation by Rad3-dependent phosphorylation. In the second stage, primed Cds1 molecules dimerize via phospho-specific interactions mediated by the FHA domains and are activated by autophosphorylation. This two-stage activation mechanism for the replication checkpoint allows for rapid activation with a high signal-to-noise ratio.

[Keywords: Cds1 autoactivation; Cds1 autophosphorylation; Cds1 dimerization; Mrc1 SQ cluster; Mrc1 TQ repeats; replication checkpoint]

Supplemental material is available at http://www.genesdev.org.

Received December 30, 2005; revised version accepted February 9, 2006.

The genome of a eukaryotic cell is duplicated with remarkable fidelity during each cell cycle (Kelly and Brown 2000; Bell and Dutta 2002). Several factors account for the extraordinary precision of this process: the intrinsic accuracy of the polymerization machinery, the efficient correction of rare polymerization errors by post-replication repair processes, and the operation of a surveillance mechanism, referred to as the replication checkpoint, that can detect and deal with perturbations that impede the normal progression of replication forks. The replication checkpoint is a complex signal transduction pathway that is activated when nucleotide pools become limiting for DNA synthesis or replication forks encounter unrepaired lesions in the template (Nyberg et al. 2002; Osborn et al. 2002; Ahn et al. 2004). Under these circumstances, checkpoint activation is crucial for maintenance of genomic integrity and cell survival. The checkpoint triggers downstream pathways that prevent futile initiation events, stimulate the repair or bypass of DNA damage, delay mitosis, and stabilize the replisome, allowing replication to resume when normal conditions

${ }^{3}$ Corresponding author.

E-MAIL tkelly@mskcc.org; FAX (646) 422-2189.

Article and publication are at http://www.genesdev.org/cgi/doi/10.1101/ gad.1406706. are restored. It has been suggested that defects in the replication checkpoint contribute to genomic instability and cancer (Nyberg et al. 2002; Bartkova et al. 2005; Gorgoulis et al. 2005).

Genetic studies, particularly in Schizosaccharomyces pombe and Saccharomyces cerevisiae, have identified several components of the replication checkpoint (Boddy and Russell 2001; Nyberg et al. 2002). In S. pombe, Rad3, a protein kinase related to the phosphoinositide 3-kinases (PIKKs), forms a complex with Rad26 and binds to DNA structures (possibly single-stranded regions) associated with stalled replication forks (Wolkow and Enoch 2002; Zou and Elledge 2003). Rad3 phosphorylates key targets to activate the replication checkpoint, including the effector kinase Cds1 (see below). A heterotrimeric ring-like complex that contains Rad9, Hus1, and Rad1 is also loaded onto chromatin at sites of stalled replication forks (Osborn et al. 2002). This so-called 9-1-1 complex is related to the replication processivity factor PCNA, and its association with DNA is dependent on the activity of a specific loader complex, containing Rad17 and Rfc2-5, that binds to single- to double-strand transitions in DNA (Venclovas and Thelen 2000; Majka and Burgers 2003). The recruitment of 9-1-1 to stalled forks is independent of Rad3-Rad26 (Kondo et al. 2001; Melo et al. 2001). While it is clear that $9-1-1$ is essential for activa- 
tion of the replication checkpoint (Lindsay et al. 1998), its precise role in the process remains unknown.

The major effector responsible for most of the biological effects of the replication checkpoint is the protein kinase Cds1, which is related to S. cerevisiae Rad53 and mammalian Chk2 (Ahn et al. 2004). Activation of Cds1 requires all of the checkpoint components described above, and the kinase is a target of Rad3-dependent phosphorylation (Tanaka et al. 2001). Work in S. pombe and $S$. cerevisiae has shown that the phosphorylation and activation of Cds1 (and Rad53) is dependent on an additional factor, Mrc1 (for mediator of the replication checkpoint). Mrc1 was discovered by genetic screens and shown to be required for viability when replication is blocked by hydroxyurea (HU) (Alcasabas et al. 2001; Tanaka and Russell 2001). It appears that Mrc1 is a component of the replisome and travels with replication forks (Katou et al. 2003; Osborn and Elledge 2003). When fork progression is blocked, Mrc1 is phosphorylated, presumably by Rad3 and Tel1, and the phosphorylation of Mrc1 is essential for activation of Cds1 (Tanaka and Russell 2004). Recent evidence strongly suggests that Mrc1 phosphorylation also contributes directly to the stabilization of stalled replication forks (Katou et al. 2003; Osborn and Elledge 2003).

Although many of the players have been identified, little is currently known about the biochemical mechanisms that lead to activation of the replication checkpoint or the role of Mrcl in this process. S. pombe represents an excellent model system to study these mechanisms because the replication checkpoint is activated by a linear pathway that converges on a single effector kinase, Cds1 (Furuya and Carr 2003). We report here that the effector of the replication checkpoint, Cds1, is activated in two stages. In the priming stage, Cds1 is recruited to stalled forks by an interaction that is dependent on phosphorylation of one of two critical Rad3 consensus sites in Mrc1. The bound Cds1 then undergoes Rad3-dependent phosphorylation. In the autoactivation stage, phospho-Cds1 is activated via dimerization and autophosphorylation by a mechanism that probably does not require the further participation of Mrc1 or Rad3. This two-stage activation mechanism for the replication checkpoint allows for rapid activation with a high signalto-noise ratio.

\section{Results}

Two clusters of potential Rad3/Tel1 phosphorylation sites in Mrc1 are required for survival in $\mathrm{HU}$

Mrc1 has been identified by two laboratories as an Sphase-specific gene required for activation of the replication checkpoint pathway in response to the inhibitor of ribonucleotide reductase, HU (Alcasabas et al. 2001; Tanaka and Russell 2001). We independently identified Mrc1 in a bioinformatics search of the $S$. pombe genome for novel genes regulated by Cdc10, a transcription factor required for the G1-S transition (Lowndes et al. 1992) and observed that Mrc1 is essential for the replication checkpoint (see Supplementary Fig. S1 for details).

Previous work has shown that Mrc1 is hyperphosphorylated when cells are treated with HU (Tanaka and Russell 2001; Zhao et al. 2003). We made use of SDSPAGE to monitor the effects of mutations in various protein kinase genes on Mrc1 hyperphosphorylation. Mrcl in extracts from wild-type cells treated with $\mathrm{HU}$ exhibited several bands of lower mobility that were not present in untreated cells (Fig. 1A). The shifted bands were sensitive to phosphatase treatment, indicating that they are the result of phosphorylation (data not shown). The pattern observed in cells deficient in Rad3 was similar to that of wild-type cells except for the absence of a band of intermediate mobility (marked with asterisks in Fig. 1A). Deletion of both the rad3 and tel1 genes completely abolished Mrcl hyperphosphorylation. These data suggest that either Tell or Rad3 can phosphorylate Mrc1, but that there are likely to be some differences in their sites of phosphorylation. Because cells lacking Tel1 are not sensitive to HU (Lindsay et al. 1998), the Rad3 phosphorylation sites must be critical for activation of the replication checkpoint. We obtained direct evidence that this is the case in experiments to be described later. Interestingly, Mrc1 was hyperphosphorylated in cells deficient in the effector kinase, Cds1, even in the absence of HU (Fig. 1A). This finding suggests that perturbations of DNA replication capable of activating the replication checkpoint pathway may occur in normal cell cycles and cause persistent checkpoint signaling when the effector kinase is absent. These data are consistent with those reported by others (Zhao et al. 2003).

Rad3 and Tel1 preferentially phosphorylate target proteins at SQ and TQ sites. S. pombe Mrc1 contains 14 such sites, including a dense cluster of eight sites located near the middle of the protein (Fig. 1B). To examine the potential function(s) of the phosphorylation sites, we changed the $S$ and $T$ residues to alanines in three groups and assayed the resulting strains for sensitivity to HU. The N-terminal group contained four SQ/TQ residues (N4), the middle group contained seven SQ/TQ residues (Mid7), and the C-terminal group contained three SQ/TQ residues (C3). Elimination of the $\mathrm{N}$-terminal and $\mathrm{C}$-terminal SQ/TQ sites had little, if any, effect on sensitivity to $\mathrm{HU}$ (Fig. 1C, upper panel). In contrast, the elimination of the Mid7 group of SQ/TQ sites resulted in a sensitivity to HU comparable to that observed when Mrcl was deleted. To determine the relative importance of the seven SQ/TQ sites in the Mid7 group, we tested the HU sensitivity of various combinations of single mutations (Fig. 1C, lower panel). We observed that simultaneous mutation of two TQ sites, T645A and T653A, resulted in HU sensitivity identical to that of the Mrcl deletion. The single mutations reduced HU sensitivity much less than the double mutation, indicating that the functions of T645 and T653 are redundant. In this regard, it is interesting to note that T645 and T653 are located in adjacent short tandem repeats (Fig. 1B, roman numerals). Mutation of T634 or S637 had no effect on HU sensitivity (data not shown). However, simultaneous mutation 
A

Figure 1. Phosphorylation of Mrc1 in two distinct domains regulates the replication checkpoint. (A) Phosphorylation of Mrcl is dependent on Rad3 and Tell. S. pombe wild-type and $\Delta \mathrm{rad} 3$ or $\Delta$ tel1 cells were treated with 25 $\mathrm{mM}$ HU. Samples were removed every hour, and the mobility of Mrcl was determined by SDS-PAGE and Western blotting. The bands of lower mobility represent phosphorylated Mrc1 (data not shown). The bands of intermediate mobility marked with asterisks are Rad3-specific (position indicated by the arrow in $\Delta$ rad 3 lanes). (B) Locations of Rad3 and Tel1 consensus phosphorylation sites in Mrcl. TQ sites are labeled in red and SQ sites in yellow. The 14 SQ/TQ sites were initially changed to AQ sites in three groups: N4, Mid7, and C3, as indicated in the top panel of $C$. Three tandem repeats of eight amino acids are indicated by roman numerals. Repeat I, which contains an SQ (S637), is cryptic and does not ordinarily contribute to checkpoint activation (see Fig. 3D). Repeats II and III, which contain TQ motifs (T645 and T653), are functionally redundant and are referred to in the text as the TQ repeats. The phosphorylation sites from S572 to $\mathrm{S} 614$ are referred to as the SQ cluster. $(C)$ The TQ repeats and the SQ cluster of Mrcl are independently required for checkpoint activation. Wild-type Mrcl and various mutants were expressed on plasmids under the control of the $\mathrm{mrc1}^{+}$promoter in $\Delta m r c 1$ cells. Fivefold dilutions of logarithmically growing cells were spotted on YE6S plates containing increasing concentrations of $\mathrm{HU}$ and incubated at $30^{\circ} \mathrm{C}$ for $3 \mathrm{~d}$.

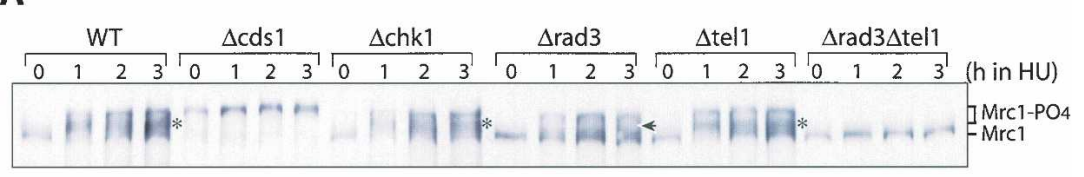

B

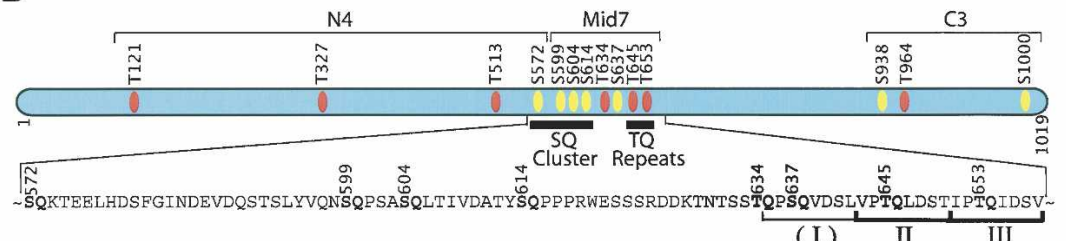

c

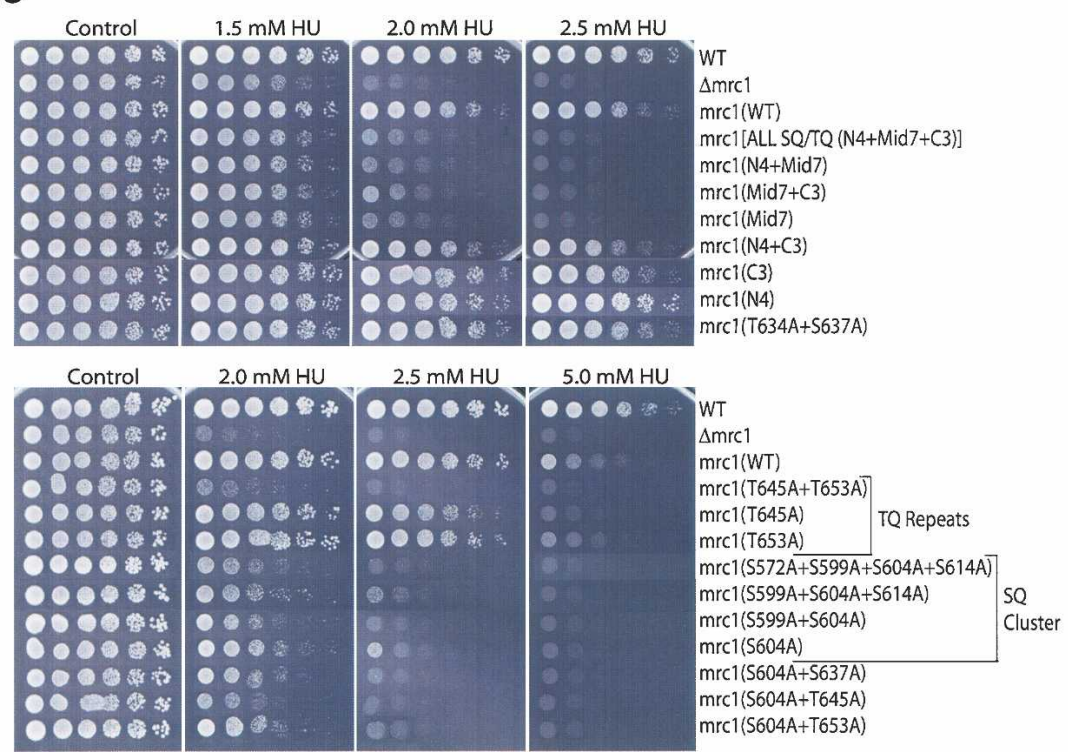

of the four sites in the SQ cluster (S572, S599, S604, and S614) resulted in sensitivity to HU almost as great as that of the Mrc1 deletion. Analysis of various combinations of SQ mutations demonstrated that S604 is most critical for Mrc1 function, but that the other SQ sites contribute as well (Fig. 1C, lower panel; data not shown). These results differ from those reported previously (Zhao et al. 2003), particularly the observation that T645 and T653 play critical, but redundant, roles in activation of the replication checkpoint. Taken together, the data indicate that the SQ/TQ sites in Mrcl have two separable, nonredundant functions that are both required for Mrc1 activity, one mediated by the T645 and T653 repeats and the other by the several SQ sites clustered in the middle region. For simplicity, we refer to the two groups as the TQ repeats and the SQ cluster, respectively (Fig. 1B).

The Mrc1 TQ repeats are required for activation of Cds1 and phosphorylation of Cds1 on T11

To further explore the functions of the TQ repeats and SQ cluster, we monitored the phosphorylation and acti- vation of the Cds1 checkpoint kinase. We observed that the T645A-T653A double mutation completely eliminated the phosphorylation and activation of Cds1 in the presence of HU (Fig. 2A). Mrc1 was hyperphosphorylated in this genetic background, consistent with continuous signaling through the checkpoint pathway in the absence of the effector kinase. The single mutants T645A and T653A exhibited only slightly reduced Cds1 activity, consistent with the redundant functions of T645 and T653 in controlling HU sensitivity. Mutations in the SQ cluster appeared to have cumulative effects on Cds1 activation. The triple mutation S599A-S604A-S614A greatly reduced, but did not completely eliminate, Cds 1 activity in the presence of HU. The single mutation S604A reduced Cds1 activation only slightly, while a double mutant (S599A-S604A) exhibited intermediate levels of Cds1 activation.

The $\mathrm{N}$ terminus of Cds1 contains three TQ/SQ sites, one of which, T11Q12, has been shown to be a target of phosphorylation by $\operatorname{Rad} 3$ and to be required for activation of Cds1 in the presence of HU (Tanaka and Russell 2004). The effect of Mrc1 TQ/SQ mutations on T11 phosphorylation was assessed by Western blotting with a 
A

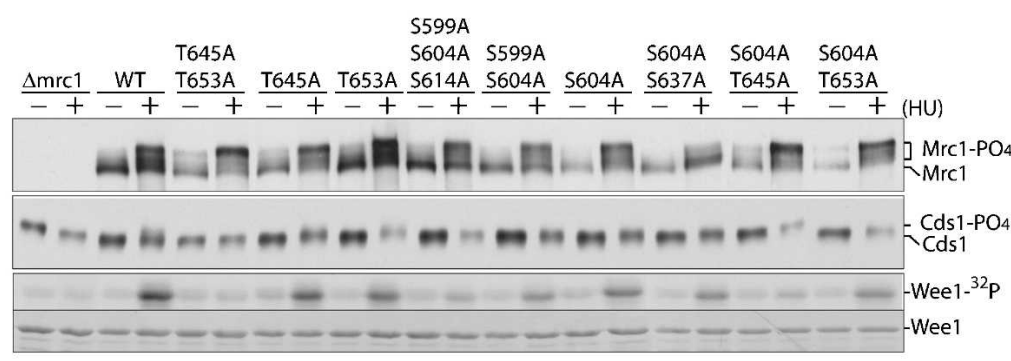

B

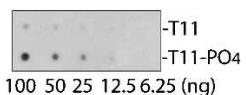

$100502512.56 .25(\mathrm{ng})$

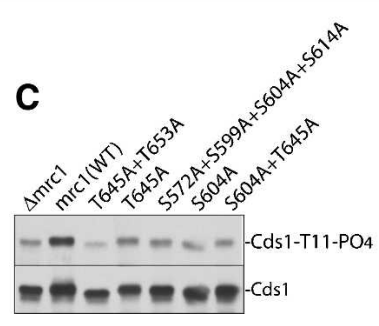

Figure 2. Phosphorylation of $\mathrm{Mrcl}$ TQ repeats is required for phosphorylation of Cds1 on T11 and activation of Cds1 protein kinase activity. (A) Mutation of both Mrc1 TQ repeats abolishes activation of Cds1. S. pombe wild-type or mutant cells were incubated in the presence $(+)$ or absence $(-)$ of 25 $\mathrm{mM} \mathrm{HU}$ for $3 \mathrm{~h}$ at $30^{\circ} \mathrm{C}$. The phosphorylation states of Mrc1 (top panel) and Cds1 (middle panel) were analyzed by SDS-PAGE and Western blotting. (Bottom panel) Cds1 kinase activity was measured with GST-Weel as substrate (Boddy et al. 1998). (B) Specificity of anti-Cds1 T11 phospho-specific antibody. Various amounts of phosphorylated and nonphosphorylated peptides (residues 2-20) containing Cds1 T11 were spotted on a nitrocellulose membrane and subjected to Western blotting with the affinity-purified T11 phospho-specific antibody. $(C)$ Mutation of both TQ repeats abolishes Cds1 phosphorylation on T11. Wild-type and mutant Mrc1 were expressed in cds1-2HA6his $\Delta m r c 1$ cells. After HU treatment, Cds1 was purified from cell extracts by immunoprecipitation with anti-HA antibody beads. The phosphorylation state of Cds1 T11 was determined by Western blotting with the purified T11 phospho-specific antibody.
T11 phospho-specific antibody (Fig. 2C). Deletion of Mrc1 eliminated T11 phosphorylation, as recently reported (Tanaka and Russell 2004). The T645A-T653A double mutation also reduced T11 phosphorylation to background. Mutation of the SQ cluster greatly reduced, but did not eliminate, T11 phosphorylation. Taken together, the data of Figure 2 indicate that the TQ repeats are absolutely required for T11 phosphorylation and subsequent activation of Cds1. The SQ cluster strongly facilitates phosphorylation and activation of Cds1 but does not appear to be absolutely essential.

\section{Phosphorylated Mrc1 TQ repeats directly bind the FHA domain of Cds1}

Cds1 contains a forkhead-associated (FHA) domain that is located just C-terminal to T11 (see Fig. 4A, below). The FHA domain of Cds1 has been shown to bind to phosphothreonine-containing peptides, but its natural targets are largely unknown (Durocher et al. 2000; Durocher and Jackson 2002). We made use of surface plasmon resonance (SPR) to determine whether the FHA domain of Cds1 is capable of interacting with the phosphorylated TQ repeats of Mrcl. For this purpose, a 15amino-acid peptide from Mrc1 (residues 645-659) containing a phospho-T653 repeat was immobilized on a BIAcore sensor chip via a biotin residue at the $\mathrm{N}$ terminus. The interaction of the peptide with the GST-tagged FHA domain from Cds1 in the mobile fluid phase was monitored by SPR. We observed that the Cds1 FHA domain bound to the T653 phosphopeptide with high affinity (Fig. 3A, left) and that the interaction was dependent on the phosphoryl group (Fig. 3A, middle). Mutations of the FHA domain that abolished activation of Cds1 (see Fig. 4) also eliminated the interaction with the
T653 phosphopeptide (Fig. 3A, right). We also tested the ability of Cds1 FHA domain to bind to a 20-amino-acid peptide from Mrc1 (residues 593-612) containing phospho-S604, which appears to be the most important residue in the SQ cluster (Fig. 1C). We did not observe any significant binding of the S604 phosphopeptide to the Cds1 FHA domain or the full-length Cds1 (data not shown). In addition, we observed that an SQ motif could not functionally replace a TQ motif (T645) in one of the TQ repeats (Fig. 3D). These data are consistent with previous observations indicating that FHA domains interact preferentially with motifs containing phosphothreonine (Durocher et al. 1999; Durocher and Jackson 2002) and strongly suggest that phosphorylated TQ repeats in Mrc1 are required to recruit Cds1 during checkpoint activation. The data further suggest that phosphorylated SQ motifs are not directly involved in binding Cds1 and presumably play a different role in checkpoint activation.

The two TQ repeats II and III have the consensus sequence I/V-P-T-Q-I/L-D-S. While a phosphorylated threonine in this sequence is clearly essential for the interaction of Mrc1 with the FHA domain of Cds1, it is likely that other residues are also involved in the interaction. Studies of the binding of the Rad53 and Cds1 FHA domains to synthetic peptide libraries have suggested that an aspartate in the +3 position relative to the phosphothreonine may be a critical contact (Durocher et al. 1999). Both TQ repeats contain an aspartate at the +3 position. Mutation of these residues to alanines (D648A, D656A) resulted in HU sensitivity comparable to that of the double mutant lacking both threonines (T645AT653A) (Fig. 3D). Immediately adjacent to the TQ repeats II and III is a homologous repeat (I) that contains SQ in place of TQ and an aspartate residue in the +3 position relative to the $\mathrm{S} 637$ (Fig. 3D). Interestingly, con- 
Figure 3. The Mrc1 TQ repeats are phosphorylated by $\operatorname{Rad} 3$ and bind to the FHA domain of Cds1. (A) Determination of the binding affinity of a phosphorylated Mrc1 TQ repeat for the FHA domain of Cds1 by SPR. Peptides containing Mrc1 T653 (biotin-TQLDSTIPTQIDSVQ, residues 645-659), with (left panel) or without (middle panel) a phosphoryl group on T653 (underlined) were adsorbed onto the surface of a streptavidin SA sensor chip to $\sim 10 \mathrm{RU}$. Various concentrations of purified GST-FHA (residues 1-179 of Cds1) were allowed to flow over the chip, and binding to the peptide was measured by SPR. The concentrations of GST-FHA in the fluid phase were $15,7.5,3.75,1.88,0.94,0.47,0.23,0.12,0.06$, $0.03,0.02$, and $0 \mu \mathrm{M}$. (Right panel) The binding of GSTFHA containing R64A and N107A mutations, which are known to abolish checkpoint activation, was also studied. $(B)$ The Mrc1 TQ repeats are phosphorylated in vivo. $S$. pombe cells expressing Mrc1-6his1HA were incubated in the presence $(+)$ or absence of $(-)$ of HU. Mrcl was affinity-purified from cell extracts with TALON resin in the presence of $6 \mathrm{M} \mathrm{GnHCl}$. (Top panel) The purified protein was subjected to SDS-PAGE, transferred to a nitrocellulose membrane, and probed with GSTFHA. The bound GST-FHA was detected by HRP-conjugated goat anti-GST antibody. The SQ cluster mutant contained S572A, S599A, S604A, and S614A. (Lower panel) Mrcl was detected by Western blotting with antiHA antibody after the GST-FHA was stripped off. $(C)$ Phosphorylation of the TQ repeats is dependent on Rad3. Mrc1 was purified from HU-treated wild-type, $\Delta m r c 1, \Delta r a d 3$, or $\Delta t e l 1$ cells and analyzed as in $B$. The

Mrc1(T645A + T653A) mutant served as a negative control. Loading of Mrc1 was normalized to wild type. $(D)$ Effects of mutations in the three Mrcl tandem repeats. The primary sequence of the three repeats and HU sensitivity of the mutants are shown in the top and lower panels, respectively. Serine substitution of T645 abolished the ability of TQ repeat II to activate the replication checkpoint (cf. T645S + T653A and T645A + T653A). Threonine substitution of S637 converted the nonfunctional repeat (I) to a partially functional repeat (cf. S637T + T645A + T653A and T645A + T653A), while threonine substitution of S604 did not. Mutation of the aspartate of the TQ repeats II and III caused HU sensitivity slightly less than that by the mutation of TQ motifs (cf. D648A, D656A, and D648A + D656A with T645A, T653A, and T645A + T653A). Insertion of two TQ repeats in a second locus between amino acids 517 and 518 partially rescued the double TQ mutation (cf. T645A + T653A and T645A + T653A + 2× rpt).

version of this SQ motif to a TQ motif partially rescued mutation of both TQ repeats (Fig. 3D; cf. Mrc1-S637TT645A-T653A and Mrc1-T645A-T653A). These data suggest that the phosphorylated TQ consensus sequence is sufficient to bind the FHA domain of Cds1. Consistent with this hypothesis, insertion of a module containing two TQ-repeat consensus sequences at a second site in Mrcl partially rescued the HU sensitivity of the T645AT653A mutant (Fig. 3D).

As a more direct test of the hypothesis that the Mrc1 TQ repeats recruit Cds1 in vivo following replication blocks, we carried out a Far Western experiment with the GST-tagged FHA domain from Cds1 as probe. For this purpose, Mrc1 was purified from $S$. pombe extract, separated by SDS-PAGE, and transferred to a nitrocellulose membrane. The GST-FHA probe recognized a band with the mobility of phospho-Mrc1 that was specifically present in extracts from HU-treated S. pombe (Fig. 3B). This band was not present in cells lacking Mrcl or in cells expressing Mrcl in which all SQ and TQ motifs were eliminated by mutation (all SQ/TQ). Importantly, GST-FHA failed to bind to Mrc1-T645A-T653A, which lacks the TQ repeats. The single mutation T645A did not eliminate GST-FHA binding, consistent with the redundancy of the two sites in checkpoint activation. When all sites in the SQ cluster were mutated, binding of GST-FHA was reduced, but not completely eliminated. The single SQ mutation, S604A, did not reduce binding of GST-FHA to Mrcl, suggesting that the effects of the SQ motifs are partially redundant. We conclude from these experiments that the TQ repeats of Mrcl play a critical role in recruiting Cds1 following an HU block. The SQ cluster of Mrcl is not absolutely required for recruitment, but plays a facilitatory role.

We also made use of the Far Western method to determine which protein kinase is required for phosphorylation of the TQ repeats in Mrc1 (Fig. 3C). We were able to detect binding of the GST-FHA probe to $\mathrm{Mrcl}$ in cells lacking Tel1, but not in cells lacking Rad3. Since Rad3 and Tell appear to be responsible for all detectable phosphorylation of Mrcl when replication is blocked by HU (Fig. 1A), this result strongly suggests that Rad3 specifically phosphorylates the Mrc1 TQ repeats, although we cannot rule out the unlikely possibility that $\operatorname{Rad} 3$ controls the activity of some other TQ-specific protein kinase. Our data provide an explanation for the observa- 
A

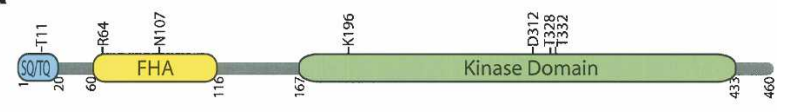

B

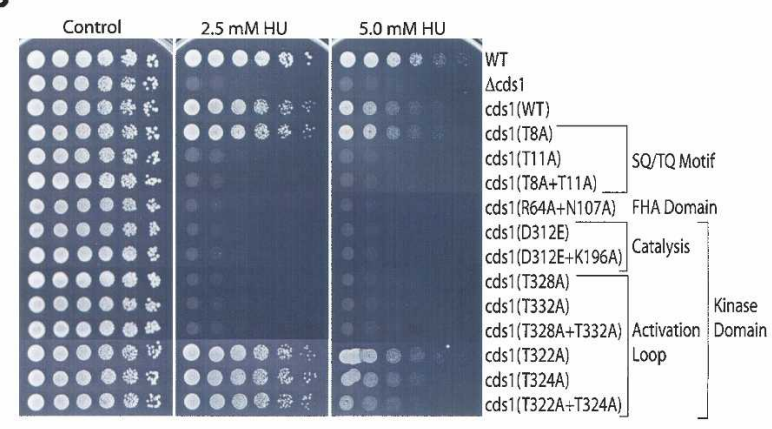

C

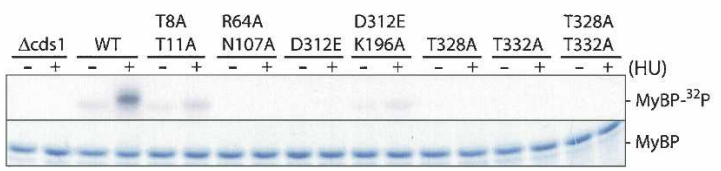

Figure 4. Analysis of the three functional domains of Cds1 by mutagenesis. (A) Diagram of the three functional domains of Cds1: SQ/TQ motifs, FHA domain, and kinase domain. The conserved essential residues analyzed by mutagenesis are marked on the top. $(B)$ HU sensitivity of Cds1 mutants. The mutants were expressed from plasmids under the control of the $c d s 1^{+}$promoter in $\Delta c d s 1$ S. pombe. HU sensitivity was tested as in Figure 1C. $(C)$ Protein kinase activity of Cds1 mutants. Wildtype or mutant Cds1 was immunoprecipitated from HU-treated $(+)$ or untreated $(-)$ cells. The activity of Cds1 was measured using MyBP as the substrate (Lindsay et al. 1998) in standard kinase buffer containing $50 \mu \mathrm{M}\left[\gamma^{-32} \mathrm{P}\right] \mathrm{ATP}$. The reaction products were subjected to SDS-PAGE. MyBP was visualized by autoradiography (top) and Coomassie blue staining (bottom).

tion that activation of Cds 1 is dependent on $\operatorname{Rad} 3$, but not Tel1 (Lindsay et al. 1998).

\section{Three domains in Cds1 are essential for Mrc1-dependent activation}

The data described above indicate that the FHA domain of Cds1 is required to recruit the kinase to Mrcl when replication forks are blocked. One consequence of this recruitment is phosphorylation of Cds1 on T11, probably by Rad3. The remaining question is how these events lead to the activation of Cds1 as a protein kinase. To begin to answer this question, we studied the effects of mutation of various conserved residues in Cds1 on HU sensitivity and activation of Cds1 kinase. As previously reported, T11 is absolutely required for activation of the replication checkpoint and kinase activity (Tanaka et al. 2001). We confirmed this result (Fig. 4B) and showed that mutation of the remaining SQ/TQ motifs in Cds1 (T8, S19, S155, and S379) did not significantly increase sensitivity to $\mathrm{HU}$ (Fig. 4B; data not shown). We also confirmed the previous report that the FHA domain is ab- solutely required for the activation of the replication checkpoint and Cds1 protein kinase (Fig. 4B,C; Tanaka and Russell 2004), consistent with the essential role of the FHA domain in recruitment of Cds1 to Mrc1. As described below, the FHA domain plays another essential role in Cds1 activation as well. Mutations in the active site of Cds1 protein kinase domain that abolish kinase activity (D312E and D312E-K196A) are sensitive to HU (Lindsay et al. 1998). We further analyzed the requirement for potential phosphorylation sites in the activation loop of Cds1. There are four such sites, but we observed that alanine substitutions of two of them, T322 and T324, have no significant effect on activation of the replication checkpoint. Substitution of T328 and T332, however, resulted in HU sensitivity equivalent to that of $\Delta c d s 1$ and abolished kinase activity, suggesting that activation loop phosphorylation may be important for Cds1 activity (Fig. 4B,C). Complementation studies in which various $c d s 1$ mutants were coexpressed demonstrated that all three motifs-T11, the FHA domain, and catalytic/activation loop residues-must be present in the same Cds1 molecule for activation of kinase activity in the presence of HU (Supplementary Fig. S3).

\section{The FHA domain of Cds1 directly binds phosphorylated Cds1 T11 and facilitates autophosphorylation}

We made use of SPR to examine the ability of a 19residue peptide containing phosphorylated T11 of Cds1 (residues 2-20) to bind to the FHA domain of Cds1 (Fig. 5A). The experimental design was essentially identical to that of Figure 3A. We observed that the Cds1 FHA domain bound to the phosphopeptide with an apparent dissociation constant of $\sim 3.8 \mu \mathrm{M}$, an affinity $\sim 10$ - to 15 fold lower than that for the Mrc1 phosphopeptide (Fig. 3A). The interaction was dependent on the phosphoryl group and was abolished by mutations in the FHA domain (Fig. 5A, middle and right panels).

We next asked whether the interaction between the FHA domain and phosphorylated T11 might promote autophosphorylation of Cds1. To explore this possibility, we purified activated Cds1 from HU-treated wild-type $S$. pombe cells and tested its ability to phosphorylate a mutant form of Cds1(D312E) lacking kinase activity. The mutant Cds1(D312E) was purified from bacteria and was present in 20 -fold excess over the activated wild-type kinase. The two proteins were incubated together in vitro under standard kinase reaction conditions and then separated by SDS-PAGE. The mutant Cds1(D312E) was an excellent substrate for the activated wild-type kinase, indicating that Cds1 is capable of efficient autophosphorylation in trans (Fig. 5B, upper left panel). Strikingly, the observed autophosphorylation was almost completely eliminated by mutations in the FHA domain of the Cds1(D312E) substrate (Fig. 5B, upper right panel). Quantification showed that the initial rate of the kinase reaction was reduced 400-fold (Fig. 5B, lower panels). These FHA mutations are the same as those shown to abolish the interaction with T11 in the SPR experiments 
Figure 5. Intermolecular interactions between the FHA domain and phosphorylated T11 of Cds 1 molecules promote autophosphorylation in trans. (A) Analysis of the binding of the Cds1 FHA domain to a Cds1 peptide containing phosphorylated T11 by SPR. SPR measurements were carried out as in Figure 3A. The immobile phase contained the Cds1 T11 peptide (biotin-EEPEEATQATQEAPLHVSQ, residues 2-20) with or without a phosphate at T11 (underlined). The fluid phase contained GST-FHA of Cds1 at the same concentrations used in the experiment of Figure 3A. (Left panel) Peptide with a phosphoryl group at T11. (Middle panel) Peptide without the phosphoryl group. (Right panel) Mutant GSTFHA(R64A + N107A). (B) Autophosphorylation of Cds1 is dependent on a functional FHA domain. Activated Cds1 kinase (90 nM) purified from HU-treated S. pombe was mixed with kinase-dead Cds1(D312E, $1875 \mathrm{nM}$ ) purified from $E$. coli as substrate in standard kinase buffer containing $100 \mu \mathrm{M}\left[\gamma^{-32} \mathrm{P}\right] \mathrm{ATP}$. The reaction was incubated at $30^{\circ} \mathrm{C}$, and aliquots were removed at various intervals. (Top panel) The substrate Cds1(D312E) was separated from the activated Cds1 kinase by SDS-PAGE on an $8 \%$ gel. (Lower panel) The incorporation of ${ }^{32} \mathrm{P}$ was quantified with PhosphorImager. (Right half) A second reaction was carried out under identical conditions except that the kinase-dead Cds1 substrate contained R46A and N107A mutations in the FHA domain.

(Fig. 5A). Thus, the data indicate that autophosphorylation is strongly facilitated by the intermolecular interaction between phosphorylated $\mathrm{T} 11$ and the FHA domain.

Since Cds1 can undergo autophosphorylation in trans, an interesting question is whether Cds1 itself can mediate T11 phosphorylation. If this were the case, it would provide a possible autoamplification mechanism. However, this possibility seems unlikely since it has been shown that the kinase-dead Cds1(D312E) mutant undergoes efficient T11 phosphorylation in vivo when cells are treated with HU (Tanaka et al. 2001), an observation that we have confirmed (data not shown). These data indicate that T11 phosphorylation in vivo is not dependent on Cds1 kinase activity and support the view that T11 phosphorylation is a function of $\operatorname{Rad} 3$. We show below that the critical targets of autophosphorylation for Cds1 activation as a protein kinase appear to lie in the catalytic domain.

\section{Concentration-dependent autoactivation of Cds1 in vitro and in vivo}

To further understand the mechanism of Cds1 activation, we purified the enzyme in its inactive form from $\Delta$ rad3 $\Delta$ tel1 cells. Increasing concentrations of the purified enzyme were incubated with ATP and a constant amount of myelin basic protein (MyBP) as substrate (Fig. 6A). Little Cds1 kinase activity was observed when the enzyme concentration was $<100 \mathrm{nM}$. Above this level, kinase activity increased dramatically with concentration and was associated with autophosphorylation /data not shown). The data were closely fit by a function in which kinase activity is proportional to the 1.97 power of the Cds 1 concentration $\left(\mathrm{R}^{2}=0.996\right)$. The striking nonlinearity of the rate of substrate phosphorylation with enzyme concentration suggested that autoactivation of Cds1 occurred during the reaction. One possibility, confirmed below, is that autoactivation was the result of Cds1 autophosphorylation in trans. We have estimated the intracellular concentration of Cds1 to be in the neighborhood of $80 \mathrm{nM}$ (Supplementary Fig. S4C). At this concentration, the activity of the enzyme would be expected to be quite low based on the in vitro data (Fig. 6A). Dimerization of Cds1 via the interaction between phosphorylated $\mathrm{T} 11$ and the FHA domain, described above, could drive Cds1 activation by increasing the rate of autophosphorylation. The results in Figure 6A suggest that the normal mechanism of activation can be bypassed by raising the enzyme concentration to nonphysiological levels.

These data are consistent with the observation that expression of Cds1 at high levels in Escherichia coli yielded active Cds1 (Fig. 6B,D). The activity of Cds1 kinase expressed in bacteria was independent of the FHA domain and T11, but was eliminated by mutations in the active site (e.g., D312E) or in activation loop residues T328 or T332. Cds1 purified from E. coli was phosphorylated (Fig. 7B; data not shown), indicating that the high concentration of enzyme in bacteria was sufficient to drive autophosphorylation. As shown in Figure 6B, active Cds1 kinase is lethal to E. coli.

Similarly, active kinase was recovered when Cds1 was expressed at very high levels under the control of the $n m t 1^{+}$promoter in $\Delta$ rad $3 \Delta m r c 1 S$. pombe (Supplementary Fig. S4). The presence of active protein kinase induced a significant elongation of the cells, indicative of cell cycle delay (Supplementary Fig. S4A). As in the case 
A

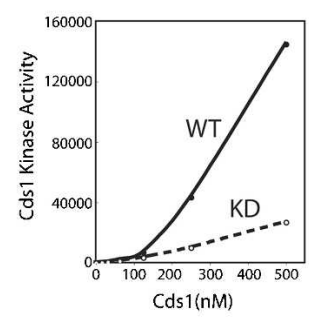

C

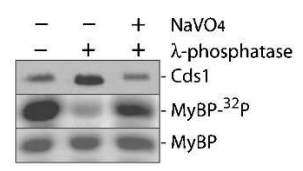

B

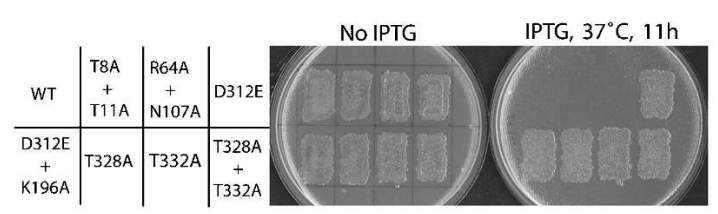

D

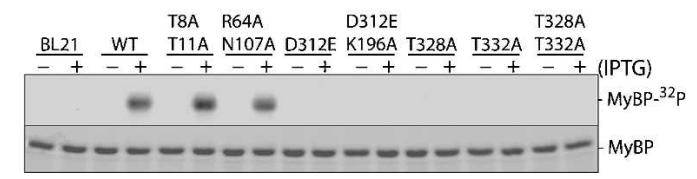

Figure 6. The weak intrinsic kinase activity of Cds1 is greatly enhanced by autophosphorylation. (A) Concentration-dependent autoactivation of Cds1 in vitro. Unactivated Cds1 and the kinase-dead Cds1 mutant (D312E) were purified from $\Delta$ rad3stel1 S. pombe and incubated in standard kinase buffer containing MyBP as the substrate. Cds1 kinase activity was measured by ${ }^{32} \mathrm{P}$ incorporation into $\mathrm{MyBP}$ and plotted against the concentrations of Cds1. (Solid line, WT) Wild-type Cds1; (dashed line, KD) kinase-dead Cds1 mutant D312E. (B) Suppression of growth of E. coli by Cds1 kinase activity. (Left plate) E. coli strains harboring vectors expressing wild-type or mutant Cds1 under the control of the lac promoter were grown on

an LB plate in the absence of IPTG. The cells were replicated onto a plate containing IPTG to induce Cds1 expression. $(C)$ The activity of activated Cds1 is dependent on phosphorylation. Cds1 was purified from HU-treated S. pombe by immunoprecipitation with anti-HA antibody beads. The beads were then split into three aliquots; the first aliquot received phosphatase buffer alone, the second received $400 \mathrm{U}$ of $\lambda$-phosphatase, and the third received the same amount of phosphatase plus $20 \mathrm{mM} \mathrm{NaVO}_{4}$. After an incubation at

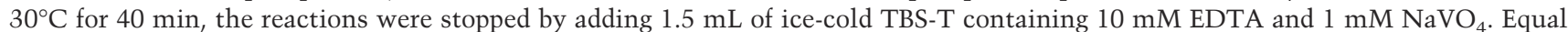
amounts of phosphatase and $\mathrm{NaVO}_{4}$ were added to the first and the second aliquots. After washing the beads five times with TBS-T containing $1 \mathrm{mM} \mathrm{NaVO}_{4}$, the kinase activity was examined in standard kinase buffer containing $1 \mathrm{mM} \mathrm{NaVO}_{4}$ and $\mathrm{MyBP}$ as the substrate. $(D)$ Activities of wild-type and mutant Cds1 expressed in E. coli. Extracts were prepared from the E. coli strains described in $B$. Wild-type or mutant Cds1 was purified by immunoprecipitation with anti-HA antibody beads. Cds1 kinase activity was measured in standard kinase buffer using MyBP as the substrate.

of E. coli, the observed in vitro kinase activity was independent of the FHA domain and T11, but was eliminated by mutations in the active site (e.g., D312E) or in activation loop residues T328 or T332 (Supplementary Fig. S4B).

To verify that the activity of Cds1 activated in vivo is dependent on phosphorylation, we tested the effect of phosphatase treatment on Cds1 kinase activity (Fig. 6C). Wild-type $S$. pombe cells were treated with $\mathrm{HU}$, and the activated Cds1 was purified by binding to HA antibody beads. Cds1 activity was greatly reduced in samples treated with phosphatase, but not in mock-treated samples or samples treated with phosphatase in the presence of the inhibitor $\mathrm{NaVO}_{4}$.

\section{Induced dimerization of the Cds1 catalytic domain promotes autophosphorylation and kinase activation in vitro}

To directly test the role of dimerization and autophosphorylation in the activation of Cds1, we made a construct encoding a chimeric enzyme consisting of an FK506-binding protein (FKBP) fused to the Cds1 catalytic domain (residues 150-460, Cds1 ${ }^{\text {cat}}$ ). This fusion construct lacks both T11 and the FHA domain, but can be dimerized by addition of the small bifunctional molecule AP20187 (Ariad) (Fig. 7A; Spencer et al. 1993). The fusion protein was expressed in E. coli and analyzed by SDSPAGE after induction for various times. After 30 min of induction, the protein migrated as a single band of the expected mobility (Fig. 7B, left panel). Most of the protein migrated in bands of lower mobility after 90 min of induction. These bands were the result of autophos- phorylation of the fusion protein in E. coli as demonstrated by (1) the absence of shifted bands when a kinasedead fusion protein (D312E) was expressed under the same conditions (Fig. 7B, right panel) and (2) the sensitivity of the shifted bands to phosphatase (Fig. 7C). To obtain the unphosphorylated form of the fusion protein for in vitro studies, we purified it from E. coli after only $30 \mathrm{~min}$ of induction and treated with phosphatase to remove any residual phosphoryl groups. When the purified protein was incubated with increasing concentrations of AP20187 and analyzed on native PAGE gels, a slower moving band, presumably consisting of the induced dimers, was observed (Fig. 7D). This band reached maximal intensity at a molar ratio of AP20187 to protein of $\sim 0.63$ and then decreased in intensity at higher concentrations of AP20187. In the presence of ATP and MyBP as substrate, both autophosphorylation and enzyme activity dramatically increased with AP20187 concentration, also reaching a maximum at a molar ratio of $\sim 0.63$ (Fig. 7E, left panels). At concentrations of AP20187 $>0.63$, autophosphorylation and kinase activity rapidly decreased as expected (Fig. 7A). As a control, the same experiments were carried out with a fusion protein containing a mutant (D312E) catalytic domain (Fig. 7E, right panels). The mutant protein formed dimers, but did not undergo autophosphorylation or activation.

To rule out the unlikely possibility that activation of the FKBP-Cds $1^{\text {cat }}$ fusion protein was due to a conformational change induced by dimerization rather than autophosphorylation, we examined the ATP dependence of the activation step in a two-stage reaction (Supplementary Fig. S5). In this experiment the purified fusion protein was first incubated in the presence or absence of 
Figure 7. Induced homodimerization of the catalytic domain of Cds1 promotes autophosphorylation and activation of kinase activity in vitro. (A) Diagram illustrating the induction of dimers of FKBP-Cds $1^{\text {cat }}$ fusion protein by the bifunctional dimerizer AP20187. The fusion protein contains an FKBP domain (black) followed by the Cds1 catalytic domain (Cds1 $1^{\text {cat }}$, residues 150-460, green). AP20187 is shown in blue. Maximum dimerization occurs at a molar ratio of AP20187 to fusion protein of 1:2. At significantly lower or higher concentrations of AP20187, the fusion protein is predominantly monomeric. (B) Expression of the FKBP-Cds $1^{\text {cat }}$ containing wild-type (WT) Cds1 or kinase-dead Cds1 (D312E) catalytic domains. Extracts of E. coli growing at $20^{\circ} \mathrm{C}$ were prepared at the indicated times after induction and analyzed by SDS-PAGE and Western blotting. $(C)$ The FKBP$\mathrm{Cds} 1^{\text {cat }}$ fusion protein is phosphorylated in E. coli. The fusion protein was purified from $E$. coli extracts after 60 min of induction and treated with buffer alone, $\lambda$-phosphatase, or $\lambda$-phosphatase plus $3.3 \mathrm{mM} \mathrm{NaVO}_{4}$ at $30^{\circ} \mathrm{C}$. (D) Purified FKBP-Cds $1^{\text {cat }}(400 \mathrm{nM})$ containing the wildtype or kinase-dead Cds1(D312E) catalytic domain was incubated for $30 \mathrm{~min}$ on ice with various concentrations of AP20187 in $10 \mu \mathrm{L}$ of buffer containing $20 \mathrm{mM}$ Tris: $\mathrm{HCl}(\mathrm{pH} 8.0)$ and $50 \mathrm{mM} \mathrm{NaCl}$. Samples were analyzed by electrophoresis on an $8 \%$ native polyacrylamide gel and stained with silver. (E) Autophosphorylation and activation of Cds1 are stimulated by induced dimerization. Purified FKBP-Cds $1^{\text {cat }}$ fusion protein (50 $\mathrm{nM}$ ) was incubated with the same concentrations of AP20187 as in $D$ in $20 \mu \mathrm{L}$ of kinase buffer containing 50 $\mu \mathrm{M}\left[\gamma^{-32} \mathrm{P}\right] \mathrm{ATP}$ and $5 \mu \mathrm{g}$ of MyBP. After incubation at $30^{\circ} \mathrm{C}$ for $20 \mathrm{~min}$, the reactions were stopped by adding 5 $\mu \mathrm{L}$ of $5 \times$ SDS gel loading buffer. The MyBP substrate and the FKBP-Cds $1^{\text {cat }}$ fusion protein were separated by a $12 \%$ SDS-PAGE. The top half of the gel, containing the FKBP-Cds $1^{\text {cat }}$ fusion protein, was transferred onto a nitrocellulose membrane for Western blotting and autoradiography. The lower half of the gel, containing the substrate MyBP, was stained with Coomassie blue to visualize MyBP. Incorporation of ${ }^{32} \mathrm{P}$ into MyBP was measured by PhosphorImager. (Right half of figure) As a control, the same experiment was performed with a fusion protein containing the Cds1(D312E) mutation in the catalytic domain.

ATP with AP20187 at a molar ratio of 0.63 to induce dimerization. Following the first incubation, a high concentration of AP20187 was added to promote dissociation of dimers, and enzyme activity was then assayed with MyBP as substrate. We observed that the activity of the enzyme preincubated with ATP (Supplementary Fig. S5, lane 4) was much greater than that of the enzyme preincubated in the absence of ATP (Supplementary Fig. S5, lane 2). Control experiments in which the AP20187 concentration was maintained at a molar ratio of 0.63 during the second incubation demonstrated that a high concentration of the compound was effective in preventing dimerization, autophosphorylation, and activation (Supplementary Fig. 5, cf. lanes 1 and 2). Taken as a whole, the data presented in Figures 6 and 7 and Supplementary Figure S5 indicate that induced dimerization is sufficient to activate Cds1 and that activation is dependent on autophosphorylation.

\section{Dimerization activates Cds1 in vivo and causes cell cycle delay}

To examine the effects of induced dimerization in vivo, we made plasmid constructs encoding full-length Cds1 with two FKBPs at the $\mathrm{N}$ terminus (2xFKBP-Cds1). (Two FKBP modules were used in order to increase the efficiency of induced dimerization.) The fusion protein was expressed in $\Delta \mathrm{rad} 3 \Delta \mathrm{cds} 1 \mathrm{~S}$. pombe under the control of the $c d s 1^{+}$promoter. In the presence of $1 \mu \mathrm{M}$ AP20187, cells expressing the fusion construct exhibited an elongated phenotype indicative of cell cycle delay (Fig. 8A). A similar phenotype has been observed when wild-type Cds1 (Supplementary Fig. S4A) or GST-tagged Cds1 (Boddy et al. 1998) is expressed at a very high level in $S$. pombe. Cell elongation was not observed in the absence of AP20187 or with untagged Cds1 or with a kinase-dead fusion construct containing the D312E mutation [2xFKBP-Cds1(D312E)]. The 2xFKBP-Cds1 fusion protein was immunoprecipitated from cell extracts and assayed for activity with MyBP as substrate (Fig. 8B). Significant kinase activity was observed in immunoprecipitates of 2xFKBP-Cds1 prepared from cells treated with AP20187, but not in those prepared from mock-treated cells nor those in immunoprecipitates of 2xFKBP-Cds1(D312E). To ensure that the observed kinase activity was independent of T11 and the FHA domain of Cds1, we made a fusion construct with two FKBP domains fused to the $\mathrm{N}$ terminus of the catalytic domain of the enzyme 
A

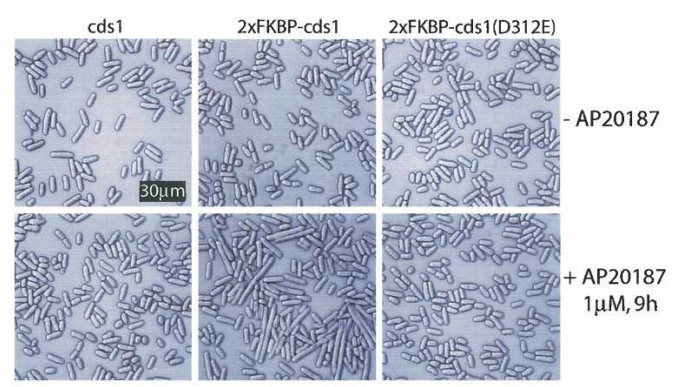

C

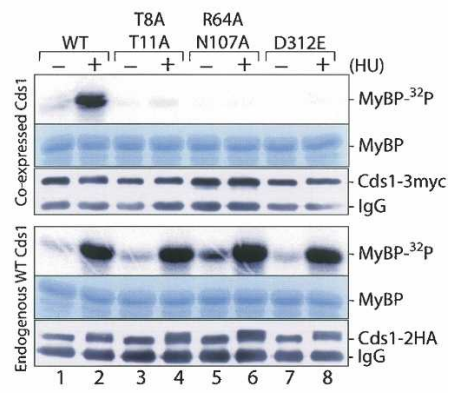

B

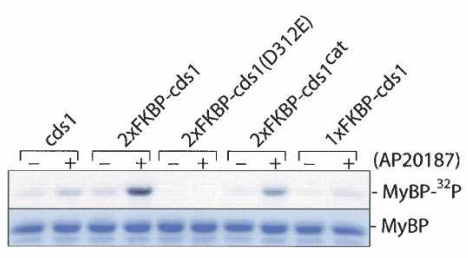

D

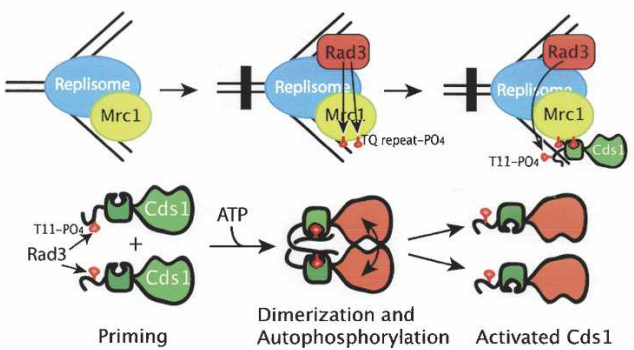

Figure 8. Dimerization of Cds1 activates Cds1 and bypasses the requirement for Rad3 in vivo. (A) Induced dimerization of Cds1 causes cell mitotic delay (cdc phenotype). S. pombe $\Delta$ rad3 $\Delta c d s 1$ cells expressing wild-type Cds1, the full-length Cds1 fused with two tandem FKBP domains at the $\mathrm{N}$ terminus (2xFKBP-Cds1), or the 2xFKBP-Cds1(D312E) fusion protein containing an inactivating mutation in the catalytic domain were grown for $9 \mathrm{~h}$ at $30^{\circ} \mathrm{C}$ in the presence of $1 \mu \mathrm{M}$ AP20187. The cells were photographed in a phasecontrast microscope. Bar, $30 \mu \mathrm{m}$. (B) Induced dimerization activates Cds1 in vivo. S. pombe $\Delta$ rad3scds1 cells carrying plasmids expressing the fusion proteins were incubated with $(+)$ or without $(-) 1 \mu \mathrm{M} \mathrm{AP2} 20187$ for $9 \mathrm{~h} \mathrm{at} 30^{\circ} \mathrm{C}$. The proteins were immunoprecipitated from cell extracts, and kinase activities were measured in a standard kinase assay using MyBP as substrate. 2xFKBP-cds1 and 2xFKBP-cds1(D312E) are described in A. 2xFKBP-cds $1^{\text {cat }}$ contains the Cds1 kinase domain (159-460 amino acids) fused to two FKBPs at its $\mathrm{N}$ terminus. 1xFKBP-cds1 contains the full-length Cds1 fused to one FKBP at the $\mathrm{N}$ terminus. $(C)$ Activated wild-type Cds1 kinase cannot activate mutant Cds1 molecules lacking Rad3 phosphorylation sites. Wild-type and various mutant Cds1 molecules (indicated at the top of the figure) were tagged with 6his3myc and coexpressed with HA-tagged wild-type Cds1 in cds1-6his2HA $S$. pombe. After incubation in the presence (+) or absence (-) of $\mathrm{HU}$, the differentially tagged Cds 1 molecules were separately purified by immunoprecipitation with anti-HA or anti-myc antibody beads. After extensive washing, Cds1 kinase activity retained on the beads was determined in standard kinase assays with MyBP as the substrate. Samples were analyzed by SDS-PAGE in a 12\% gel, and the incorporated ${ }^{32} \mathrm{P}$ was detected by autoradiography $\left(\mathrm{MyBP}_{-}{ }^{32} \mathrm{P}\right)$. The upper half of the figure shows the kinase activity of the myc-tagged Cds1, while the lower half shows the kinase activity of the endogenous HA-tagged Cds1. The immunoprecipitated Cds1 protein in each sample was detected by Western blotting (Cds1-3myc or Cds1-2HA), and the MyBP substrate was detected by Coomassie blue staining (MyBP). (D) Model for the two-stage activation of Cds1. Mrc1 moves along with the replisome under normal conditions (Katou et al. 2003; Osborn and Elledge 2003; Zhao and Russell 2004). When replication forks are stalled, Rad3 is recruited to the stalled forks and phosphorylates the TQ repeats of Mrc1. Either one of the two phosphorylated TQ repeats of Mrc1 can recruit an inactive Cds1 by binding to its FHA domain. Rad3 then phosphorylates T11 of the recruited Cds1, priming Cds1 for autoactivation. The phosphorylated T11 of the primed Cds1 binds to another primed Cds1 molecule, bringing the two inactive kinase domains in close proximity. In the presence of ATP, the dimerized kinase domains can autophosphorylate each other in trans, thus activating Cds1.

(2xFKBP-Cds $\left.1^{\text {cat }}\right)$. This construct could also be activated in vivo by treatment with AP20187 (Fig. 8B).

\section{Efficient dimerization and activation of Cds1 in vivo requires Rad3-dependent phosphorylation of both monomers}

The results described above demonstrate that dimerization of Cds1 can promote autophosphorylation and activation of the kinase. We have also shown that Cds1 dimerization can be driven by the interaction of phosphorylated T11 of one molecule with the FHA domain of another molecule. In principle, a Cds1 dimer could be held together by either one or two such interactions, depending on whether one or both partners have undergone Rad3-dependent phosphorylation on T11. These two possibilities have different implications for checkpoint activation in vivo. If a single phosphoT11-FHA interaction is sufficient to drive dimerization and autoactivation under in vivo conditions, it follows that Rad3dependent phosphorylation of a few Cds1 molecules might be sufficient to activate many unphosphorylated Cds1 molecules, resulting in autoamplification of the checkpoint signal. On the other hand, if two phosphoT11-FHA interactions are required for dimerization and autoactivation, then only Cds1 molecules that have been phosphorylated by $\operatorname{Rad} 3$ can be activated, so autoamplification would not be possible. To distinguish 
between these possibilities, we examined the ability of wild-type Cds1 to activate a mutant form of Cds1 (T8AT11A) that cannot be phosphorylated by Rad3 (Fig. 8C). For this purpose, wild-type or mutant Cds1 genes (tagged with myc) were coexpressed with an endogenous wildtype Cds1 gene (tagged with HA). The cells were treated with $\mathrm{HU}$ and the differentially tagged Cds1 molecules were separately purified by immunoprecipitation with the appropriate antibodies. The kinase activities of the purified proteins were measured in the standard assay with $\mathrm{MyBP}$ as substrate. In all experiments the endogenous Cds1-HA exhibited robust activation following HU treatment (Fig. 8C, lower half). When wild-type Cds1-myc was coexpressed in the same cell, it too exhibited HU-dependent activation (Fig. 8C, upper panel, lanes 1,2). However, when the coexpressed Cds1-myc contained mutations (T8A-T11A) that prevent Rad3-dependent phosphorylation, only slight activation of the mutant kinase was observed (Fig. 8C, upper panel, lanes $3,4)$. As expected, Cds1 with inactivating mutations in the FHA domain (R64A-N107A) or the catalytic domain (D312E) was not activated following HU treatment (Fig. 8C, upper panel, lanes 5-8). These data indicate that active Cds1 that has been phosphorylated by Rad3 cannot efficiently activate unphosphorylated Cds1. Thus, under physiological conditions, it appears that Cds1 dimerization that leads to kinase activation requires that both partners have undergone Rad3-dependent phosphorylation on T11. This requirement for two independent phosphorylation events may serve to increase the noise immunity of the replication checkpoint and is consistent with the observation that $\operatorname{Rad} 3$ is required for initiation and maintenance of the checkpoint signaling during HU block (Martinho et al. 1998).

\section{Discussion}

We have shown that activation of the replication checkpoint in S. pombe occurs in two stages (Fig. 8D). In the first stage, the effector kinase Cds1 is primed for autoactivation. A key step in this process is the Rad3-dependent phosphorylation of the adaptor Mrcl at sites in the TQ repeats and the SQ cluster. The phosphorylated TQ repeats function as specific docking sites for the FHA domain of Cds1, recruiting the latter to the vicinity of Rad3. This interaction is facilitated in an unknown fashion by the phosphorylated SQ motifs. Once bound to phospho-Mrc1, Cds1 undergoes Rad3-dependent phosphorylation at T11. In the second stage, Cds1 is activated via autophosphorylation, probably in the activation loop. This process is dependent on dimerization of Cds1 via interactions between phosphorylated T11 motifs and the FHA domains.

In our model, Mrcl functions only in the first stage of checkpoint activation and is not required for Cds1 autoactivation. In this view, autophosphorylation and activation of Cds1 occur after dissociation from Mrc1. While this is the simplest version of the model and consistent with all the data, we cannot rule out the possibility that
Mrc1 may facilitate autoactivation via additional interactions with Cds1.

The model in Figure 8D is consistent with the specificity and noise immunity required for the replication checkpoint. Specificity is provided by the requirement for two different protein-protein interactions to activate Cds1: the phospho-Mrc1-Cds1 interaction in the first stage and the phospho-Cds1-phospho-Cds1 interaction in the second stage. The noise immunity of the activation reaction derives from the fact that autophosphorylation of Cds1 proceeds at a very low rate in the absence of dimerization under physiological conditions (Fig. 5). Thus, little autophosphorylation/activation can take place without the priming reaction.

We have shown that the priming reaction depends on an interaction between the FHA domain of Cds1 and one of two redundant phosphopeptide repeats in Mrc1. The specificity of binding likely depends on additional interactions besides the FHA-phosphothreonine contact. Consistent with previous studies using synthetic peptide libraries (Durocher et al. 2000), we have demonstrated the importance of the aspartate at the +3 position of the Mrc1 TQ repeats in the activation of the replication checkpoint. The Cds1-docking repeats on Mrc1 described here have not been identified for Mrc1 or Rad9 of budding yeast and are clearly different from those of Xenopus Claspin. The Chk1-binding motifs on Xenopus Claspin are probably not phosphorylated by the Rad3like kinases ATR and ATM (Kumagai and Dunphy 2003). We have also shown that phosphorylation of the SQ cluster in Mrc1 facilitates the recruitment of Cds1. Since a phosphorylated SQ motif does not have significant affinity for the FHA domain of Cds1 or full-length Cds1, the effect of the SQ motifs is likely indirect. One possibility is that phosphorylation of the SQ cluster induces a conformational change in Mrcl that permits more efficient binding or phosphorylation of Cds1 by Rad3.

Our data provide strong evidence that primed Cds1 is activated via dimerization and autophosphorylation of residues in the catalytic domain. First, the activity of Cds1 purified from HU-treated S. pombe is completely dependent on phosphorylation. Second, at high protein concentration (micromolar or greater), autoactivation (and autophosphorylation) of highly purified Cds1 occurs in vitro in the absence of other factors. Third, autoactivation of Cds1 can be induced in vitro and in vivo by enforced dimerization of a fusion construct lacking both T11 and the FHA domain. Fourth, autoactivation of the Cds1 fusion construct requires both dimerization and the presence of ATP. It is likely that the critical targets of autophosphorylation reside in the activation loop of Cds1 (Nolen et al. 2004). Cds1 and HsChk2 have sequence similarities to protein kinases that require phosphorylation of the activation loop for full activity. Mutations that eliminate potential phosphorylation sites in the activation loop of Cds1 (T328 and T332) prevent activation of the kinase. These two sites correspond to residues T383 and T387 in HsChk2 that have been shown to be essential for kinase activity (Lee and Chung 2001).

The adaptor Mrcl is the key factor in the S. pombe 
replication checkpoint pathway, ensuring that cells respond to replication blocks and activate the correct downstream effector, Cds1. There is evidence in S. cerevisiae that Mrc1 may be a component of the replisome and travel along DNA with the chain elongation machinery (Katou et al. 2003; Osborn and Elledge 2003). However, this has not yet been confirmed in other systems. How cells recognize stalled replication forks is not clear. One possibility is that uncoupling of DNA unwinding from DNA synthesis may occur when nucleotides are scarce or the fork encounters an unrepaired lesion. Uncoupling may result in the generation of longlived structures containing single- to double-strand transitions that can bind $\operatorname{Rad} 3-\operatorname{Rad} 26$ and trigger the loading of the 9-1-1 complex (Bermudez et al. 2003; Ellison and Stillman 2003; Majka and Burgers 2003; Zou and Elledge 2003; Byun et al. 2005). In this scenario, the simple proximity of $\operatorname{Rad} 3$ to Mrc1 at stalled forks results in Mrc1 phosphorylation and initiation of the checkpoint cascade. Alternatively, Mrcl may play some more direct role in recognition of stalled replication forks as suggested by studies in S. cerevisiae. Further work will be required to distinguish these possibilities.

Besides serving as an adaptor in the replication checkpoint, Mrc1 may have additional roles in DNA replication. In S. cerevisiae (Osborn and Elledge 2003), deletion of Mrc1 results in greater sensitivity to acute replication blocks and DNA damage than elimination of the Mrc1 TQ and SQ motifs. We have observed a similar phenomenon in S. pombe (Supplementary Fig. S2). Thus, activation of the effector kinase Cds1 may not be the only means by which $\mathrm{Mrcl}$ protects cells from the effects of perturbations of DNA replication.

The role of Rad9 in the activation of $S$. cerevisiae Rad53 in response to DNA damage may be similar to the role of Mrc1 in the replication checkpoint described here. Earlier work had suggested that multimeric Rad9 complexes recruit Rad53 molecules, thus increasing the local concentration and allowing Rad53 autophosphorylation in trans (Gilbert et al. 2001). This model is quite different from our model for Mrc1 function. Recent work, however, is consistent with the alternative hypothesis that a major function of Rad9 is to recruit Rad53, enabling its phosphorylation and activation by Mec1 (Sweeney et al. 2005). The activity of Rad9, like that of Mrc1, appears to require an interaction with Rad53 that is dependent on phosphorylation and the Rad53 FHA domains. It is not yet understood in detail how Mec1 phosphorylation of Rad53 leads to its activation. In the case of Cds 1 it is clear that Rad3 phosphorylation on $\mathrm{T} 11$ is not sufficient for activation, since a second Rad3-independent phosphorylation of the catalytic domain is also required. Our data show that Mrc1dependent phosphorylation of $\mathrm{T} 11$ by $\mathrm{Rad} 3$ acts indirectly by promoting dimerization and autophosphorylation of Cds1. The same may be true for Rad9-mediated Mec1 phosphorylation of Rad53.

As noted above, there are significant similarities between the activation of Cds1 and the activation of HsChk2. There is good evidence that HsChk2 is activated via autophosphorylation of activation loop residues and that this process is mediated via HsChk2 oligomerization (Ahn and Prives 2002; Ahn et al. 2002; Xu et al. 2002). Like Cds1, both N-terminal TQ residues and the FHA domain of Chk2 are required for activation, but at present the adaptor mediating activation is unknown.

A major unsolved problem is the identification of the critical targets of the replication checkpoint. Checkpoint activation has several effects during $S$ phase, including inhibition of initiation of DNA replication, stabilization of stalled replication forks, and activation of repair/tolerance pathways. It is not yet completely clear what Cds 1 substrates mediate these effects. In S. pombe, Hsk1 and Dfp1, the homologs of S. cerevisiae Cdc7 and its regulatory subunit Dbf4, are substrates for Cds1 in vitro and in vivo (Snaith et al. 2000; Duncker and Brown 2003). Since Cdc7 kinase is absolutely required for initiation of DNA replication, its phosphorylation may contribute to the suppression of futile initiation events. The nuclease Mus81 and the recombinational repair factor Rad60 have also been identified as targets of Cds1dependent phosphorylation, and both have been shown to play a role in tolerance to perturbations of DNA replication (Boddy et al. 2001, 2003). It seems likely that additional targets of Cds 1 that contribute to stabilization of the replisome remain to be identified.

\section{Materials and methods}

Growth of $S$. pombe strains and preparation of growth media followed standard methods (Moreno et al. 1991). The following strains were generated for this study: YJ15, $\Delta \operatorname{mrc1}:: \mathrm{ura}^{+}$; YJ66, $\Delta m r c 1:: u_{r a 4}{ }^{+}$cds1-2HA6his::ura4 ${ }^{+} ;$YJ293, $\Delta m r c 1:: u r a 4^{+}$

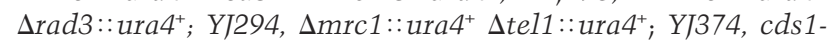
6his2HA::ura $4^{+}$. All strains contain the auxotrophic markers leu1-32, ura4-D18, ade6-M210, or ade6-M216.

Point mutations of Mrcl and Cds1 were made by QuickChange mutagenesis PCR. To examine drug sensitivity, $2 \times 10^{7}$ cells/mL of logarithmically growing $S$. pombe were diluted in fivefold steps and spotted onto YE6S or EMM6S plates that contained HU or MMS. The plates were incubated at $30^{\circ} \mathrm{C}$ for $3 \mathrm{~d}$.

Cds1 was purified as described in the Supplemental Material, and Cds1 kinase assays were performed in standard kinase buffer (20 mM Tris:HCl at pH 7.5, $5 \mathrm{mM} \mathrm{MgCl}_{2}, 1 \mathrm{mM}$ DTT, 75 $\left.\mathrm{mM} \mathrm{KCl}, 50 \mu \mathrm{M}\left[\gamma^{-}{ }^{32} \mathrm{P}\right] \mathrm{ATP}\right)$ with GST-Wee1(1-152 amino acids) (Boddy et al. 1998) or MyBP (Lindsay et al. 1998) as substrates.

Other methods used in the study such as SPR, Western, FarWestern, and purification of Cds1 are described in detail in the Supplemental Material.

\section{Acknowledgments}

We thank the members of the Kelly laboratory for many useful suggestions and J. Petrini and T. Usui for sharing preliminary results. We also thank K. Marians and B. Lang for advice on BIAcore analysis; M. Pletcher for help with RT-PCR analysis; and J. Boeke, C. Greider, and J. Corden for advice and suggestions. The Micro-Chemistry, DNA Sequencing, and Flow Cytometry, core facilities of Sloan-Kettering Institute, provided outstanding technical support for this work. We gratefully acknowledge T. Carr, P. Russell, G. Brown, S. Forsburg, and F. 
Ishikawa for yeast strains and plasmids and Ariad Pharmaceuticals, Inc., for the ARGENT Regulated Homodimerization Kit (http://www.ariad.com/regulationkits).

\section{References}

Ahn, J. and Prives, C. 2002. Checkpoint kinase 2 (Chk2) monomers or dimers phosphorylate Cdc25C after DNA damage regardless of threonine 68 phosphorylation. J. Biol. Chem. 277: 48418-48426.

Ahn, J.Y., Li, X., Davis, H.L., and Canman, C.E. 2002. Phosphorylation of threonine 68 promotes oligomerization and autophosphorylation of the Chk2 protein kinase via the forkhead-associated domain. J. Biol. Chem. 277: 19389-19395.

Ahn, J., Urist, M., and Prives, C. 2004. The Chk2 protein kinase. DNA Repair (Amst.) 3: 1039-1047.

Alcasabas, A.A., Osborn, A.J., Bachant, J., Hu, F., Werler, P.J., Bousset, K., Furuya, K., Diffley, J.F., Carr, A.M., and Elledge, S.J. 2001. Mrc1 transduces signals of DNA replication stress to activate Rad53. Nat. Cell Biol. 3: 958-965.

Bartkova, J., Horejsi, Z., Koed, K., Kramer, A., Tort, F., Zieger, K., Guldberg, P., Sehested, M., Nesland, J.M., Lukas, C., et al. 2005. DNA damage response as a candidate anti-cancer barrier in early human tumorigenesis. Nature 434: 864-870.

Bell, S.P. and Dutta, A. 2002. DNA replication in eukaryotic cells. Annu. Rev. Biochem. 71: 333-374.

Bermudez, V.P., Lindsey-Boltz, L.A., Cesare, A.J., Maniwa, Y., Griffith, J.D., Hurwitz, J., and Sancar, A. 2003. Loading of the human 9-1-1 checkpoint complex onto DNA by the checkpoint clamp loader hRad17-replication factor C complex in vitro. Proc. Natl. Acad. Sci. 100: 1633-1638.

Boddy, M.N. and Russell, P. 2001. DNA replication checkpoint. Curr. Biol. 11: R953-R956.

Boddy, M.N., Furnari, B., Mondesert, O., and Russell, P. 1998. Replication checkpoint enforced by kinases Cds1 and Chk1. Science 280: 909-912.

Boddy, M.N., Gaillard, P.H., McDonald, W.H., Shanahan, P., Yates III, J.R., and Russell, P. 2001. Mus81-Emel are essential components of a Holliday junction resolvase. Cell 107: 537-548.

Boddy, M.N., Shanahan, P., McDonald, W.H., Lopez-Girona, A., Noguchi, E., Yates, I.J., and Russell, P. 2003. Replication checkpoint kinase Cds1 regulates recombinational repair protein Rad60. Mol. Cell. Biol. 23: 5939-5946.

Byun, T.S., Pacek, M., Yee, M.C., Walter, J.C., and Cimprich, K.A. 2005. Functional uncoupling of MCM helicase and DNA polymerase activities activates the ATR-dependent checkpoint. Genes \& Dev. 19: 1040-1052.

Duncker, B.P. and Brown, G.W. 2003. Cdc7 kinases (DDKs) and checkpoint responses: Lessons from two yeasts. Mutat. Res. 532: 21-27.

Durocher, D. and Jackson, S.P. 2002. The FHA domain. FEBS Lett. 513: 58-66.

Durocher, D., Henckel, J., Fersht, A.R., and Jackson, S.P. 1999. The FHA domain is a modular phosphopeptide recognition motif. Mol. Cell 4: 387-394.

Durocher, D., Taylor, I.A., Sarbassova, D., Haire, L.F., Westcott, S.L., Jackson, S.P., Smerdon, S.J., and Yaffe, M.B. 2000. The molecular basis of FHA domain:phosphopeptide binding specificity and implications for phospho-dependent signaling mechanisms. Mol. Cell 6: 1169-1182.

Ellison, V. and Stillman, B. 2003. Biochemical characterization of DNA damage checkpoint complexes: Clamp loader and clamp complexes with specificity for $5^{\prime}$ recessed DNA. PLoS Biol. 1: E33.
Furuya, K. and Carr, A.M. 2003. DNA checkpoints in fission yeast. J. Cell Sci. 116: 3847-3848.

Gilbert, C.S., Green, C.M., and Lowndes, N.F. 2001. Budding yeast Rad9 is an ATP-dependent Rad53 activating machine. Mol. Cell 8: 129-136.

Gorgoulis, V.G., Vassiliou, L.V., Karakaidos, P., Zacharatos, P., Kotsinas, A., Liloglou, T., Venere, M., Ditullio, R.A., Kastrinakis, N.G., Levy, B., et al. 2005. Activation of the DNA damage checkpoint and genomic instability in human precancerous lesions. Nature 434: 907-913.

Katou, Y., Kanoh, Y., Bando, M., Noguchi, H., Tanaka, H., Ashikari, T., Sugimoto, K., and Shirahige, K. 2003. S-phase checkpoint proteins Tof 1 and Mrc1 form a stable replication-pausing complex. Nature 424: 1078-1083.

Kelly, T.J. and Brown, G.W. 2000. Regulation of chromosome replication. Annu. Rev. Biochem. 69: 829-880.

Kondo, T., Wakayama, T., Naiki, T., Matsumoto, K., and Sugimoto, K. 2001. Recruitment of Mec1 and Ddc1 checkpoint proteins to double-strand breaks through distinct mechanisms. Science 294: 867-870.

Kumagai, A. and Dunphy, W.G. 2003. Repeated phosphopeptide motifs in Claspin mediate the regulated binding of Chk1. Nat. Cell Biol. 5: 161-165.

Lee, C.H. and Chung, J.H. 2001. The hCds1 (Chk2)-FHA domain is essential for a chain of phosphorylation events on hCds1 that is induced by ionizing radiation. J. Biol. Chem. 276: 30537-30541.

Lindsay, H.D., Griffiths, D.J., Edwards, R.J., Christensen, P.U., Murray, J.M., Osman, F., Walworth, N., and Carr, A.M. 1998. S-phase-specific activation of Cds1 kinase defines a subpathway of the checkpoint response in Schizosaccharomyces pombe. Genes \& Dev. 12: 382-395.

Lowndes, N.F., McInerny, C.J., Johnson, A.L., Fantes, P.A., and Johnston, L.H. 1992. Control of DNA synthesis genes in fission yeast by the cell-cycle gene cdc10+. Nature 355: 449453.

Majka, J. and Burgers, P.M. 2003. Yeast Rad17/Mec3/Ddc1: A sliding clamp for the DNA damage checkpoint. Proc. Nat1. Acad. Sci. 100: 2249-2254.

Martinho, R.G., Lindsay, H.D., Flaggs, G., DeMaggio, A.J., Hoekstra, M.F., Carr, A.M., and Bentley, N.J. 1998. Analysis of Rad3 and Chk1 protein kinases defines different checkpoint responses. EMBO J. 17: 7239-7249.

Melo, J.A., Cohen, J., and Toczyski, D.P. 2001. Two checkpoint complexes are independently recruited to sites of DNA damage in vivo. Genes \& Dev. 15: 2809-2821.

Moreno, S., Klar, A., and Nurse, P. 1991. Molecular genetic analysis of fission yeast Schizosaccharomyces pombe. Methods Enzymol. 194: 795-823.

Nolen, B., Taylor, S., and Ghosh, G. 2004. Regulation of protein kinases; Controlling activity through activation segment conformation. Mol. Cell 15: 661-675.

Nyberg, K.A., Michelson, R.J., Putnam, C.W., and Weinert, T.A. 2002. Toward maintaining the genome: DNA damage and replication checkpoints. Annu. Rev. Genet. 36: 617-656.

Osborn, A.J. and Elledge, S.J. 2003. Mrc1 is a replication fork component whose phosphorylation in response to DNA replication stress activates Rad53. Genes \& Dev. 17: 17551767.

Osborn, A.J., Elledge, S.J., and Zou, L. 2002. Checking the fork: The DNA-replication stress-response pathway. Trends Cell Biol. 12: 509-516.

Snaith, H.A., Brown, G.W., and Forsburg, S.L. 2000. Schizosaccharomyces pombe Hsklp is a potential cdslp target required for genome integrity. Mol. Cell. Biol. 20: 7922-7932.

Spencer, D.M., Wandless, T.J., Schreiber, S.L., and Crabtree, 
D.R. 1993. Controlling signal transduction with synthetic ligands. Science 262: 1019-1024.

Sweeney, F.D., Yang, F., Chi, A., Shabanowitz, J., Hunt, D.F., and Durocher, D. 2005. Saccharomyces cerevisiae Rad9 acts as a Mec1 adaptor to allow Rad53 activation. Curr. Biol. 15: 1364-1375.

Tanaka, K. and Russell, P. 2001. Mrc1 channels the DNA replication arrest signal to checkpoint kinase Cds1. Nat. Cell Biol. 3: 966-972.

- 2004. Cds1 phosphorylation by Rad3-Rad26 kinase is mediated by Forkhead-associated domain interaction with Mrc1. J. Biol. Chem. 279: 32079-32086.

Tanaka, K., Boddy, M.N., Chen, X.B., McGowan, C.H., and Russell, P. 2001. Threonine-11, phosphorylated by Rad3 and atm in vitro, is required for activation of fission yeast checkpoint kinase Cds1. Mol. Cell. Biol. 21: 3398-3404.

Venclovas, C. and Thelen, M.P. 2000. Structure-based predictions of Rad1, Rad9, Hus1 and Rad17 participation in sliding clamp and clamp-loading complexes. Nucleic Acids Res. 28: 2481-2493.

Wolkow, T.D. and Enoch, T. 2002. Fission yeast Rad26 is a regulatory subunit of the Rad3 checkpoint kinase. Mol. Biol. Cell 13: 480-492.

$\mathrm{Xu}, \mathrm{X}$., Tsvetkov, L.M., and Stern, D.F. 2002. Chk2 activation and phosphorylation-dependent oligomerization. Mol. Cell. Biol. 22: 4419-4432.

Zhao, H. and Russell, P. 2004. DNA binding domain in the replication checkpoint protein Mrcl of Schizosaccharomyces pombe. J. Biol. Chem. 279: 53023-53027.

Zhao, H., Tanaka, K., Nogochi, E., Nogochi, C., and Russell, P. 2003. Replication checkpoint protein $\mathrm{Mrcl}$ is regulated by Rad3 and Tell in fission yeast. Mol. Cell. Biol. 23: 83958403.

Zou, L. and Elledge, S.J. 2003. Sensing DNA damage through ATRIP recognition of RPA-ssDNA complexes. Science 300: 1542-1548. 


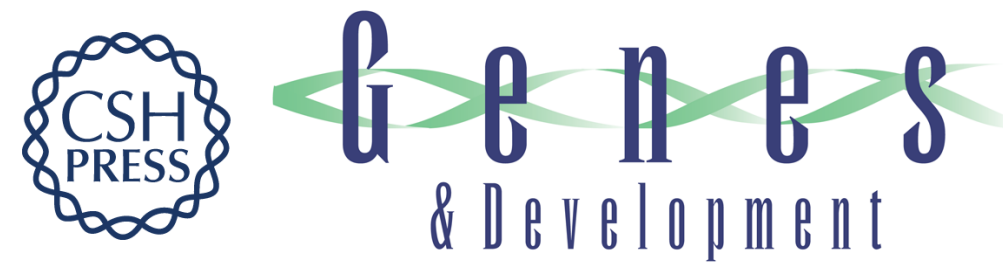

\section{Two-stage mechanism for activation of the DNA replication checkpoint kinase Cds1 in fission yeast}

Yong Xu, Matthew Davenport and Thomas J. Kelly

Genes Dev. 2006, 20:

Access the most recent version at doi:10.1101/gad.1406706

Supplemental http://genesdev.cshlp.org/content/suppl/2006/04/06/20.8.990.DC1
Material

References This article cites 47 articles, 23 of which can be accessed free at: http://genesdev.cshlp.org/content/20/8/990.full.html\#ref-list-1

License

Email Alerting Receive free email alerts when new articles cite this article - sign up in the box at the top Service right corner of the article or click here.

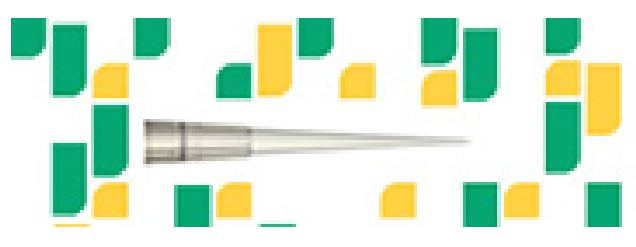

Focused on your science. 